\title{
45 Warum Übergewicht zum Typ-2-Diabetes führen kann
}

\author{
(C) Springer-Verlag GmbH Deutschland, ein Teil von Springer Nature 2018 \\ D. Mathias, Fit und gesund von 1 bis Hundert \\ https://doi.org/10.1007/978-3-662-56307-6_45
}

In Europa leiden nach Angaben der WHO (2017) ca. 60 Millionen Erwachsene an Typ-2-Diabetes, weltweit wird diese Zahl auf etwa 422 Millionen geschätzt. In Deutschland sind dies knapp 7 Millionen Menschen mit fast 300.000 Neuerkrankungen jährlich. Global verursacht diese Massenerkrankung Kosten von rund 1,3 Billionen US\$ pro Jahr (Bommer et al. 2017).

Warum Übergewicht hierfür der Hauptverursacher ist, lässt sich gut durch die Kenntnisse der dabei zugrunde liegenden molekularen Mechanismen erklären. So erzeugen der in den überschüssigen Fettablagerungen gebildete Tumornekrosefaktor $\boldsymbol{\alpha}$ und das Peptidhormon Resistin sowie das von verfetteten Lebern abgegebene Fetuin-A in den Muskelzellen eine Insulinresistenz, indem sie die Insulinrezeptoren auf den Zelloberflächen blockieren. Die Insulinresistenz führt zusammen mit einer

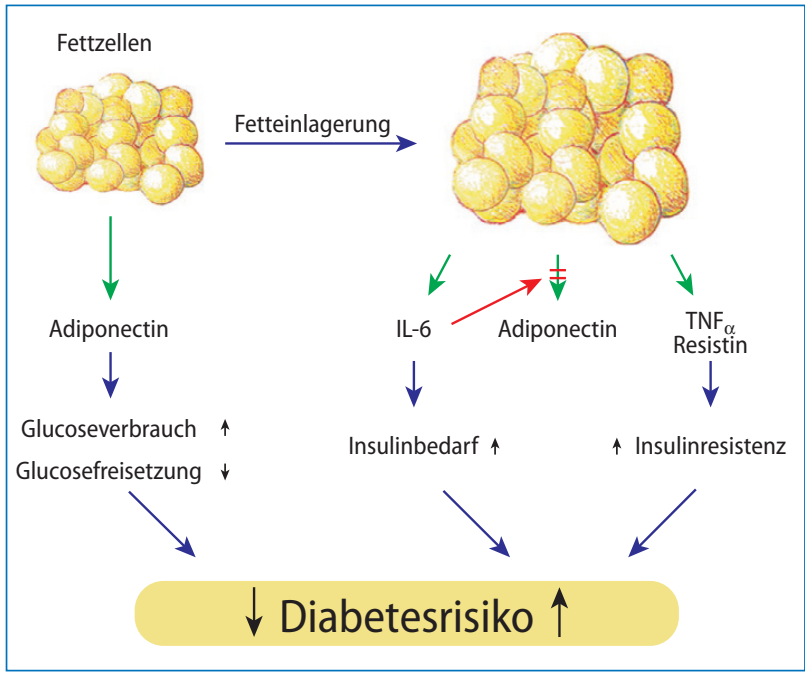

- Abb. 45.1 Molekularer Mechanismus der Entwicklung eines Typ-2Diabetes. TNFa Tumornekrosefaktor a, IL-6 Interleukin 6 dann nach längerer Zeit meist auch verminderten Insulinproduktion der $\beta$-Zellen in der Bauchspeicheldrüse zum Typ-2-Diabetes.

Der Typ-2-Diabetes ist Schrittmacher für kardiovaskuläre Erkrankungen, Krebs und Altersdemenz.

Interleukin 6, bei chronischen Entzündungen und Übergewicht auch vermehrt im Fettgewebe gebildet, ist ein weiterer Risikofaktor, weil es auch in den Leberzellen zu einer Insulinresistenz führen kann. Dagegen wurde Adiponectin bisher als Schutzfaktor betrachtet, denn höhere Spiegel von Adiponectin finden sich oft, wenn keine Erkrankung an Typ-2-Diabetes vorliegt. Das scheint zunächst auch sinnvoll, denn Adiponectin stimuliert die insulinunabhängige Glucoseverbrennung in den Muskelzellen und hemmt die Glucosefreisetzung in der Leber. Tatsächlich besteht hier aber kein kausaler Zusammenhang. Gezielte Anhebungen des Adiponectinspiegels verringern nicht das Risiko, an Typ-2-Diabetes zu erkranken (Yaghootkar et al. 2013). Das tun aber Ernährungsumstellungen und körperliche Aktivitäten (Amer. Diab. Assoc. 2017).

Bereits eine 10-prozentige Gewichtsreduktion verbessert die Insulinempfindlichkeit deutlich. Durch andauernde Muskelarbeit ist die Störung der regelhaften Ausprägung der Insulinrezeptoren auf den Muskelzellen schnell reversibel. Viel Bewegung erhöht dort auch die Zahl der Insulinrezeptoren, das Diabetesrisiko sinkt. 